\title{
Stabilization of Integral-Equation Formulations for the Accurate Solution of Scattering Problems Involving Low-Contrast Dielectric Objects
}

\author{
Özgür Ergül, Student Member, IEEE, and Levent Gürel, Senior Member, IEEE
}

\begin{abstract}
The solution of scattering problems involving low-contrast dielectric objects with three-dimensional arbitrary shapes is considered. Using the traditional forms of the surface integral equations, scattered fields cannot be calculated accurately if the contrast of the object is low. Therefore, we consider the stabilization of the formulations by extracting the nonradiating parts of the equivalent currents. We also investigate various types of stable formulations and show that accuracy can be improved systematically by eliminating the identity terms from the integral-equation kernels. Traditional and stable formulations are compared, not only for small scatterers but also for relatively large problems solved by employing the multilevel fast multipole algorithm. Stable and accurate solutions of dielectric contrasts as low as $10^{-4}$ are demonstrated on problems involving more than 250000 unknowns.
\end{abstract}

Index Terms-Dielectrics, electromagnetic scattering, multilevel fast multipole algorithm (MLFMA), surface integral equations.

\section{INTRODUCTION}

OURFACE integral equations are commonly used to formulate scattering problems involving dielectric objects with arbitrary shapes [1]. By using equivalent electric and magnetic currents, boundary conditions are satisfied on the surface of the scatterer. In the literature, there are various integral-equation formulations derived by using different combinations of the boundary conditions, testing schemes, and scaling operations [1]-[6]. Some of these formulations are known to be stable and provide accurate results when they are used to formulate dielectric objects with moderate dielectric parameters. However, these formulations tend to be less accurate as the contrast of the object decreases and the electric properties of the inner and outer media converge to each other. There are various applications that involve scattering from low-contrast objects. Examples are red blood cells in blood plasma [6], [7], plastic mines buried in soil [8], polymeric materials, such as sub-micron latex particles in water [9], and dielectric photonic crystals [10]. When

Manuscript received May 20, 2007; revised November 6, 2007. This work was supported in part by the Scientific and Technical Research Council of Turkey (TUBITAK) under Research Grant 105E172, in part by the Turkish Academy of Sciences in the framework of the Young Scientist Award Program (LG/TUBA-GEBIP/2002-1-12), and in part by contracts from ASELSAN and SSM.

The authors are with the Department of Electrical and Electronics Engineering and the Computational Electromagnetics Research Center (BiLCEM), Bilkent University, TR-06800, Bilkent, Ankara, Turkey (e-mail: ergul@ee.bilkent.edu.tr; lgurel@bilkent.edu.tr).

Color versions of one or more of the figures in this paper are available online at http://ieeexplore.ieee.org.

Digital Object Identifier 10.1109/TAP.2008.916971 the contrast is low, however, it becomes difficult to obtain accurate results with the conventional surface formulations unless a stabilization procedure is applied.

In this paper, we consider stabilization of the dielectric formulations for the accurate solution of scattering problems involving low-contrast dielectric objects. Stabilization is achieved by extracting the nonradiating parts of the equivalent currents and solving only the radiating currents as the unknowns of the problem [11]. We apply stabilization procedures to both the tangential (T) and the normal (N) formulations [5]. For large dielectric objects, scattering problems are solved iteratively with the accelerated matrix-vector multiplications by the multilevel fast multipole algorithm (MLFMA) [4]. By stabilizing the surface formulations, accuracy is regained without a significant cost in terms of processing time and memory usage.

To discretize various surface formulations, we employ RaoWilton-Glisson (RWG) [12] functions defined on triangular domains. For the same discretization of a scattering problem, the $\mathrm{T}$ formulations usually provide more accurate results compared to the $\mathrm{N}$ formulations. The inaccuracy of the $\mathrm{N}$ formulations has been extensively investigated for perfectly conducting objects [13]-[17] and moderate-contrast dielectric scatterers [5] in relation to the discretization order. Another explanation for the same inaccuracy can be based on the existence of the well-tested identity terms in the $\mathrm{N}$ formulations [18]. Using a Galerkin scheme, the $\mathrm{T}$ formulations do not contain any dominant identity terms. Therefore, they provide more accurate results at the cost of the decreased convergence rate of the iterative solutions. In this paper, we show that the stable $\mathrm{T}$ formulations are more accurate compared to the stable $\mathrm{N}$ formulations. In addition, we also consider a different stabilization procedure to further improve the accuracy of the stable $\mathrm{T}$ formulations by carefully eliminating the identity terms arising in the straightforward stabilization procedure. We demonstrate the accuracy of various formulations for the solution of the scattering problems involving sphere and cube geometries with various contrasts.

\section{SuRface Formulations OF DiElECTRIC PROBLEMS}

To construct the surface integral equations, the operators for the outside $(l=1)$ and inside $(l=2)$ the object are defined as

$$
\begin{aligned}
\mathcal{T}_{l}\{\boldsymbol{X}(\boldsymbol{r})\} & =i k_{l} \int_{S} d \boldsymbol{r}^{\prime}\left[\boldsymbol{X}\left(\boldsymbol{r}^{\prime}\right)+\frac{1}{k_{l}^{2}} \nabla^{\prime} \cdot \boldsymbol{X}\left(\boldsymbol{r}^{\prime}\right) \nabla\right] g_{l}\left(\boldsymbol{r}, \boldsymbol{r}^{\prime}\right) \\
\mathcal{K}_{l}\{\boldsymbol{X}(\boldsymbol{r})\} & =\int_{\mathrm{PV}, S} d \boldsymbol{r}^{\prime} \boldsymbol{X}\left(\boldsymbol{r}^{\prime}\right) \times \nabla^{\prime} g_{l}\left(\boldsymbol{r}, \boldsymbol{r}^{\prime}\right) \\
\mathcal{I}\{\boldsymbol{X}(\boldsymbol{r})\} & =\boldsymbol{X}(\boldsymbol{r})
\end{aligned}
$$


where PV indicates the principal value of the integral, $X$ is either the equivalent electric current $(\boldsymbol{J})$ or the equivalent magnetic current $(\boldsymbol{M})$ defined on the surface of the dielectric object $S, k_{l}$ is the wavenumber associated with medium $l$, and $g_{l}\left(\boldsymbol{r}, \boldsymbol{r}^{\prime}\right)$ denotes the homogeneous-space Green's function defined as

$$
g_{l}\left(\boldsymbol{r}, \boldsymbol{r}^{\prime}\right)=\frac{\exp \left(i k_{l} R\right)}{4 \pi R} \quad\left(R=\left|\boldsymbol{r}-\boldsymbol{r}^{\prime}\right|\right) .
$$

When the tangential components of the fields are directly sampled (tested) on the surface, the $\mathrm{T}$ formulations are derived as

$$
\begin{array}{r}
\hat{\boldsymbol{t}} \cdot\left[\begin{array}{cc}
\mathcal{Z}_{11}^{T}(a, b) & \mathcal{Z}_{12}^{T}(a, b)+\mathcal{I}_{12}^{T}(a, b) \\
\mathcal{Z}_{21}^{T}(c, d)+\mathcal{I}_{21}^{T}(c, d) & \mathcal{Z}_{22}^{T}(c, d)
\end{array}\right] \\
\cdot\left[\begin{array}{c}
\boldsymbol{J}(\boldsymbol{r}) \\
\boldsymbol{M}(\boldsymbol{r})
\end{array}\right]=-\hat{\boldsymbol{t}} \cdot\left[\begin{array}{c}
a \eta_{1}^{-1} \boldsymbol{E}^{i}(\boldsymbol{r}) \\
c \eta_{1} \boldsymbol{H}^{i}(\boldsymbol{r})
\end{array}\right]
\end{array}
$$

where the operators are defined as

$$
\begin{aligned}
& \mathcal{Z}_{11}^{T}(a, b)=\mathcal{Z}_{22}^{T}(a, b)=a \mathcal{I}_{1}+b \mathcal{I}_{2} \\
& \mathcal{Z}_{12}^{T}(a, b)=-a \eta_{1}^{-1} \mathcal{K}_{1}-b \eta_{2}^{-1} \mathcal{K}_{2} \\
& \mathcal{Z}_{21}^{T}(c, d)=c \eta_{1} \mathcal{K}_{1}+d \eta_{2} \mathcal{K}_{2} \\
& \mathcal{I}_{12}^{T}(a, b)=0.5\left(b \eta_{2}^{-1}-a \eta_{1}^{-1}\right) \hat{\boldsymbol{n}} \times \mathcal{I} \\
& \mathcal{I}_{21}^{T}(c, d)=0.5\left(c \eta_{1}-d \eta_{2}\right) \hat{\boldsymbol{n}} \times \mathcal{I}
\end{aligned}
$$

and $\{a, b, c, d\}$ represent the combination parameters. In (5)-(10), $\boldsymbol{E}^{i}(\boldsymbol{r})$ and $\boldsymbol{H}^{i}(\boldsymbol{r})$ are the incident electric and magnetic fields, $\eta_{l}$ is the impedance of the medium $l, \hat{t}$ is any tangential vector on the surface, and $\hat{\boldsymbol{n}}$ is the outward normal vector. Among various $\mathrm{T}$ formulations, the tangential Poggio-Miller-Chang-Harrington-Wu-Tsai (T-PMCHWT) [1] formulation and the combined $\mathrm{T}$ formulation (CTF) [5] are well known and commonly used in the literature. Both of these formulations are free of the internal-resonance problem and provide accurate results for moderate-contrast dielectric objects.

Unlike the $\mathrm{T}$ formulations, the $\mathrm{N}$ formulations are written as

$$
\begin{array}{r}
\hat{\boldsymbol{n}} \times\left[\begin{array}{cc}
\mathcal{Z}_{11}^{N}(a, b)+\mathcal{I}_{11}^{N}(a, b) & \mathcal{Z}_{12}^{N}(a, b) \\
\mathcal{Z}_{21}^{N}(c, d) & \mathcal{Z}_{22}^{N}(c, d)+\mathcal{I}_{22}^{N}(c, d)
\end{array}\right] \\
\cdot\left[\begin{array}{c}
\boldsymbol{J}(\boldsymbol{r}) \\
\boldsymbol{M}(\boldsymbol{r})
\end{array}\right]=-\hat{\boldsymbol{n}} \times\left[\begin{array}{c}
a \boldsymbol{H}^{i}(\boldsymbol{r}) \\
-c \boldsymbol{E}^{i}(\boldsymbol{r})
\end{array}\right]
\end{array}
$$

by using a cross product with the outward normal vector to obtain the tangential field components on the surface of the scatterer. The operators in (11) are defined as

$$
\begin{aligned}
& \mathcal{Z}_{11}^{N}(a, b)=\mathcal{Z}_{22}^{N}(a, b)=a \mathcal{K}_{1}-b \mathcal{K}_{2} \\
& \mathcal{Z}_{12}^{N}(a, b)=a \eta_{1}^{-1} \mathcal{T}_{1}-b \eta_{2}^{-1} \mathcal{T}_{2} \\
& \mathcal{Z}_{21}^{N}(c, d)=-c \eta_{1} \mathcal{T}_{1}+d \eta_{2} \mathcal{T}_{2} \\
& \mathcal{I}_{11}^{N}(a, b)=\mathcal{I}_{22}^{N}(a, b)=0.5(a+b) \hat{\boldsymbol{n}} \times \mathcal{I} .
\end{aligned}
$$

Among various $\mathrm{N}$ formulations, the combined $\mathrm{N}$ formulation (CNF) [5] and the N Müller formulation (NMF) [19] are free of the internal-resonance problem and extensively used to formulate the scattering problems of dielectric objects.
Both $\mathrm{T}$ and $\mathrm{N}$ formulations become inaccurate when the contrast of the dielectric object decreases. This breakdown is due to the large nonradiating parts of the equivalent currents on the scatterer [11]. Equivalent electric and magnetic currents defined on the surface of an object can be decomposed as

$$
\begin{gathered}
\boldsymbol{J}(\boldsymbol{r})=\hat{\boldsymbol{n}} \times \boldsymbol{H}(\boldsymbol{r})=\hat{\boldsymbol{n}} \times \boldsymbol{H}^{i}(\boldsymbol{r})+\hat{\boldsymbol{n}} \times \boldsymbol{H}^{r}(\boldsymbol{r}) \\
\boldsymbol{M}(\boldsymbol{r})=-\hat{\boldsymbol{n}} \times \boldsymbol{E}(\boldsymbol{r})=-\hat{\boldsymbol{n}} \times \boldsymbol{E}^{i}(\boldsymbol{r})-\hat{\boldsymbol{n}} \times \boldsymbol{E}^{r}(\boldsymbol{r})
\end{gathered}
$$

where $\left\{\hat{\boldsymbol{n}} \times \boldsymbol{H}^{i}(\boldsymbol{r}),-\hat{\boldsymbol{n}} \times \boldsymbol{E}^{i}(\boldsymbol{r})\right\}$ do not radiate. However, when the contrast of the object is small, these nonradiating currents dominate the total currents and the radiating currents, i.e., $\{\hat{\boldsymbol{n}} \times$ $\left.\boldsymbol{H}^{r}(\boldsymbol{r}),-\hat{\boldsymbol{n}} \times \boldsymbol{E}^{r}(\boldsymbol{r})\right\}$, are numerically insignificant compared to the nonradiating currents. As a consequence, scattered fields cannot be calculated accurately using the traditional forms of the surface integral equations. For accurate solutions, surface formulations should be modified by extracting the known nonradiating currents from the total currents and considering only the radiating currents as the unknowns of the problem [11], [20].

\section{StABILIZATION OF SURFACE FormUlations}

In this paper, we investigate the stabilization of both $\mathrm{T}$ and $\mathrm{N}$ formulations for the solution of low-contrast dielectric objects. Considering $\{a, b, c, d\}=\{1,1,1,1\}$ in (11) and extracting the nonradiating parts of the currents, we obtain a stable CNF $(\mathrm{S}-\mathrm{CNF})$ as

$$
\begin{aligned}
-\left[\begin{array}{cc}
\mathcal{I} & 0 \\
0 & \mathcal{I}
\end{array}\right] \cdot\left[\begin{array}{c}
\hat{\boldsymbol{n}} \times \boldsymbol{H}^{r}(\boldsymbol{r}) \\
-\hat{\boldsymbol{n}} \times \boldsymbol{E}^{r}(\boldsymbol{r})
\end{array}\right] \\
+\hat{\boldsymbol{n}} \times\left[\begin{array}{ll}
\mathcal{Z}_{11}^{N}(1,1) & \mathcal{Z}_{12}^{N}(1,1) \\
\mathcal{Z}_{21}^{N}(1,1) & \mathcal{Z}_{22}^{N}(1,1)
\end{array}\right] \cdot\left[\begin{array}{c}
\hat{\boldsymbol{n}} \times \boldsymbol{H}^{r}(\boldsymbol{r}) \\
-\hat{\boldsymbol{n}} \times \boldsymbol{E}^{r}(\boldsymbol{r})
\end{array}\right] \\
=-\hat{\boldsymbol{n}} \times\left[\begin{array}{ll}
\mathcal{Z}_{11}^{N}(1,1) & \mathcal{Z}_{12}^{N}(1,1) \\
\mathcal{Z}_{21}^{N}(1,1) & \mathcal{Z}_{22}^{N}(1,1)
\end{array}\right] \cdot\left[\begin{array}{c}
\hat{\boldsymbol{n}} \times \boldsymbol{H}^{i}(\boldsymbol{r}) \\
-\hat{\boldsymbol{n}} \times \boldsymbol{E}^{i}(\boldsymbol{r})
\end{array}\right] .
\end{aligned}
$$

When the contrast goes to zero, i.e., $\mu_{2} \rightarrow \mu_{1}$ and $\epsilon_{2} \rightarrow \epsilon_{1}$, $\mathrm{S}-\mathrm{CNF}$ reduces to

$$
\left[\begin{array}{cc}
\mathcal{I} & 0 \\
0 & \mathcal{I}
\end{array}\right] \cdot\left[\begin{array}{c}
\hat{\boldsymbol{n}} \times \boldsymbol{H}^{r}(\boldsymbol{r}) \\
-\hat{\boldsymbol{n}} \times \boldsymbol{E}^{r}(\boldsymbol{r})
\end{array}\right]=\left[\begin{array}{l}
0 \\
0
\end{array}\right]
$$

and the radiating currents become zero.

Considering $\{a, b, c, d\}=\left\{\eta_{1}, \eta_{2}, \eta_{2}, \eta_{1}\right\}$ in (5), applying a stabilization procedure, and using the identity

$$
\begin{aligned}
\left\{\begin{array}{c}
\hat{\boldsymbol{t}} \cdot \\
\hat{\boldsymbol{n}} \times
\end{array}\right\}\left[\begin{array}{cc}
\eta_{1} \mathcal{T}_{1} & -\mathcal{K}_{1} \\
\mathcal{K}_{1} & \eta_{1}^{-1} \mathcal{T}_{1}
\end{array}\right] \cdot\left[\begin{array}{c}
\hat{\boldsymbol{n}} \times \boldsymbol{H}^{i}(\boldsymbol{r}) \\
-\hat{\boldsymbol{n}} \times \boldsymbol{E}^{i}(\boldsymbol{r})
\end{array}\right] \\
=-0.5\left\{\begin{array}{c}
\hat{\boldsymbol{t}} \cdot \\
\hat{\boldsymbol{n}} \times
\end{array}\right\}\left[\begin{array}{c}
\boldsymbol{E}^{i}(\boldsymbol{r}) \\
\boldsymbol{H}^{i}(\boldsymbol{r})
\end{array}\right]
\end{aligned}
$$

we obtain a stable CTF (S-CTF) as

$$
\begin{aligned}
& \hat{\boldsymbol{t}} \cdot\left[\begin{array}{ll}
\mathcal{Z}_{11}^{T}\left(\eta_{1}, \eta_{2}\right) & \mathcal{Z}_{12}^{T}\left(\eta_{1}, \eta_{2}\right) \\
\mathcal{Z}_{21}^{T}\left(\eta_{2}, \eta_{1}\right) & \mathcal{Z}_{22}^{T}\left(\eta_{2}, \eta_{1}\right)
\end{array}\right] \cdot\left[\begin{array}{c}
\hat{\boldsymbol{n}} \times \boldsymbol{H}^{r}(\boldsymbol{r}) \\
-\hat{\boldsymbol{n}} \times \boldsymbol{E}^{r}(\boldsymbol{r})
\end{array}\right] \\
&=\hat{\boldsymbol{t}} \cdot\left[\begin{array}{ll}
\mathcal{Z}_{11}^{T}\left(\eta_{1},-\eta_{2}\right) & \mathcal{Z}_{12}^{T}\left(\eta_{1},-\eta_{2}\right) \\
\mathcal{Z}_{21}^{T}\left(\eta_{2},-\eta_{1}\right) & \mathcal{Z}_{22}^{T}\left(\eta_{2},-\eta_{1}\right)
\end{array}\right] \cdot\left[\begin{array}{c}
\hat{\boldsymbol{n}} \times \boldsymbol{H}^{i}(\boldsymbol{r}) \\
-\hat{\boldsymbol{n}} \times \boldsymbol{E}^{i}(\boldsymbol{r})
\end{array}\right]
\end{aligned}
$$


The identity in (20) is proved in the Appendix. When the contrast goes to zero, $\mathrm{S}-\mathrm{CTF}$ reduces to

$$
\hat{\boldsymbol{t}} \cdot\left[\begin{array}{cc}
\eta_{1} \mathcal{T}_{1} & -\mathcal{K}_{1} \\
\eta_{1}^{2} \mathcal{K}_{1} & \eta_{1} \mathcal{T}_{1}
\end{array}\right] \cdot\left[\begin{array}{c}
\hat{\boldsymbol{n}} \times \boldsymbol{H}^{r}(\boldsymbol{r}) \\
-\hat{\boldsymbol{n}} \times \boldsymbol{E}^{r}(\boldsymbol{r})
\end{array}\right]=\left[\begin{array}{l}
0 \\
0
\end{array}\right]
$$

and the solution is again zero. We note that CTF considered in this paper is slightly different from the original CTF derived in [5], where the combination parameters are taken as $\{a, b, c, d\}=\{1,1,1,1\}$ and the resulting integral equation contains weakly-tested identity terms.

For the simultaneous discretization of the integral equations and the scatterer surface, unknown current densities are expanded in a series of RWG functions, i.e.,

$$
\begin{aligned}
\boldsymbol{J}(\boldsymbol{r}) & =\sum_{n=1}^{N} x_{n} \boldsymbol{b}_{n}(\boldsymbol{r})=\sum_{n=1}^{N} x_{n}^{i} \boldsymbol{b}_{n}(\boldsymbol{r})+\sum_{n=1}^{N} x_{n}^{r} \boldsymbol{b}_{n}(\boldsymbol{r}) \\
\boldsymbol{M}(\boldsymbol{r}) & =\sum_{n=1}^{N} y_{n} \boldsymbol{b}_{n}(\boldsymbol{r})=\sum_{n=1}^{N} y_{n}^{i} \boldsymbol{b}_{n}(\boldsymbol{r})+\sum_{n=1}^{N} y_{n}^{r} \boldsymbol{b}_{n}(\boldsymbol{r}) .
\end{aligned}
$$

In (23)-(24), $x_{n}$ and $y_{n}$ are two sets of unknown coefficients, which are decomposed as $x_{n}=x_{n}^{i}+x_{n}^{r}$ and $y_{n}=x_{n}^{i}+y_{n}^{r}$, respectively, where $x_{n}^{i}$ and $y_{n}^{i}$ are the coefficients expanding the incident fields, i.e.,

$\hat{\boldsymbol{n}} \times \boldsymbol{H}^{i}(\boldsymbol{r})=\sum_{n=1}^{N} x_{n}^{i} \boldsymbol{b}_{n}(\boldsymbol{r})$ and $-\hat{\boldsymbol{n}} \times \boldsymbol{E}^{i}(\boldsymbol{r})=\sum_{n=1}^{N} y_{n}^{i} \boldsymbol{b}_{n}(\boldsymbol{r})$.

We note that only $\left\{x_{n}^{r}, y_{n}^{r}\right\}$ contribute to the scattered fields.

By using the method of moments with a Galerkin scheme, testing functions are also chosen as the RWG functions. For $\mathrm{S}-\mathrm{CNF}, 2 N \times 2 N$ matrix equations are derived as

$$
\begin{aligned}
-\left[\begin{array}{ll}
\overline{\boldsymbol{I}} & 0 \\
0 & \overline{\boldsymbol{I}}
\end{array}\right] \cdot\left[\begin{array}{l}
\boldsymbol{x}^{r} \\
\boldsymbol{y}^{r}
\end{array}\right]+\left[\begin{array}{ll}
\overline{\boldsymbol{Z}}_{11}^{N} & \overline{\boldsymbol{Z}}_{12}^{N} \\
\overline{\boldsymbol{Z}}_{21}^{N} & \overline{\boldsymbol{Z}}_{22}^{N}
\end{array}\right] \cdot\left[\begin{array}{l}
\boldsymbol{x}^{r} \\
\boldsymbol{y}^{r}
\end{array}\right] \\
=-\left[\begin{array}{ll}
\overline{\boldsymbol{Z}}_{11}^{N} & \overline{\boldsymbol{Z}}_{12}^{N} \\
\overline{\boldsymbol{Z}}_{21}^{N} & \overline{\boldsymbol{Z}}_{22}^{N}
\end{array}\right] \cdot\left[\begin{array}{l}
\boldsymbol{x}^{i} \\
\boldsymbol{y}^{i}
\end{array}\right]
\end{aligned}
$$

where

$$
\begin{aligned}
\overline{\boldsymbol{Z}}_{11}^{N}[m, n]= & \overline{\boldsymbol{Z}}_{22}^{N}[m, n]=\left\langle\boldsymbol{t}_{m}(\boldsymbol{r}), \hat{\boldsymbol{n}} \times \mathcal{K}_{1}\left\{\boldsymbol{b}_{n}(\boldsymbol{r})\right\}\right\rangle \\
& -\left\langle\boldsymbol{t}_{m}(\boldsymbol{r}), \hat{\boldsymbol{n}} \times \mathcal{K}_{2}\left\{\boldsymbol{b}_{n}(\boldsymbol{r})\right\}\right\rangle \\
\overline{\boldsymbol{Z}}_{12}^{N}[m, n]= & \eta_{1}^{-1}\left\langle\boldsymbol{t}_{m}(\boldsymbol{r}), \hat{\boldsymbol{n}} \times \mathcal{T}_{1}\left\{\boldsymbol{b}_{n}(\boldsymbol{r})\right\}\right\rangle \\
& -\eta_{2}^{-1}\left\langle\boldsymbol{t}_{m}(\boldsymbol{r}), \hat{\boldsymbol{n}} \times \mathcal{T}_{2}\left\{\boldsymbol{b}_{n}(\boldsymbol{r})\right\}\right\rangle \\
\overline{\boldsymbol{Z}}_{21}^{N}[m, n]= & -\eta_{1}\left\langle\boldsymbol{t}_{m}(\boldsymbol{r}), \hat{\boldsymbol{n}} \times \mathcal{T}_{1}\left\{\boldsymbol{b}_{n}(\boldsymbol{r})\right\}\right\rangle \\
& +\eta_{2}\left\langle\boldsymbol{t}_{m}(\boldsymbol{r}), \hat{\boldsymbol{n}} \times \mathcal{T}_{2}\left\{\boldsymbol{b}_{n}(\boldsymbol{r})\right\}\right\rangle \\
\overline{\boldsymbol{I}}[m, n]= & \left\langle\boldsymbol{t}_{m}(\boldsymbol{r}), \boldsymbol{b}_{n}(\boldsymbol{r})\right\rangle .
\end{aligned}
$$

In the above, the $\bar{I}$ matrix denotes the discretized form of the identity operator defined in (3). With the choice of the RWG functions for discretization, the $\overline{\boldsymbol{I}}$ matrix becomes highly sparse, but not exactly an identity matrix. ${ }^{1}$

\footnotetext{
${ }^{1}$ The $\bar{I}$ matrix could be made strictly an identity matrix if an orthonormal set of functions was used for discretization. In this paper, both the operator $\mathcal{I}$ in (3) and the matrix $\bar{I}$ in (30) are loosely referred to as "identity terms."
}

Similarly, matrix equations for S-CTF can be derived as

$$
\left[\begin{array}{ll}
\overline{\boldsymbol{Z}}_{11}^{T} & \overline{\boldsymbol{Z}}_{12}^{T} \\
\overline{\boldsymbol{Z}}_{21}^{T} & \overline{\boldsymbol{Z}}_{22}^{T}
\end{array}\right] \cdot\left[\begin{array}{l}
\boldsymbol{x}^{r} \\
\boldsymbol{y}^{r}
\end{array}\right]=\left[\begin{array}{ll}
\overline{\boldsymbol{Y}}_{11}^{T} & \overline{\boldsymbol{Y}}_{12}^{T} \\
\overline{\boldsymbol{Y}}_{21}^{T} & \overline{\boldsymbol{Y}}_{22}^{T}
\end{array}\right] \cdot\left[\begin{array}{l}
\boldsymbol{x}^{i} \\
\boldsymbol{y}^{i}
\end{array}\right]
$$

where

$$
\begin{aligned}
\overline{\boldsymbol{Z}}_{11}^{T}[m, n]= & \eta_{1}\left\langle\boldsymbol{t}_{m}(\boldsymbol{r}), \mathcal{T}_{1}\left\{\boldsymbol{b}_{n}(\boldsymbol{r})\right\}\right\rangle \\
& +\eta_{2}\left\langle\boldsymbol{t}_{m}(\boldsymbol{r}), \mathcal{T}_{2}\left\{\boldsymbol{b}_{n}(\boldsymbol{r})\right\}\right\rangle \\
\overline{\boldsymbol{Z}}_{12}^{N}[m, n]= & -\left\langle\boldsymbol{t}_{m}(\boldsymbol{r}), \mathcal{K}_{1}\left\{\boldsymbol{b}_{n}(\boldsymbol{r})\right\}\right\rangle \\
& -\left\langle\boldsymbol{t}_{m}(\boldsymbol{r}), \mathcal{K}_{2}\left\{\boldsymbol{b}_{n}\right\}\right\rangle \\
\overline{\boldsymbol{Z}}_{21}^{N}[m, n]= & \eta_{1} \eta_{2}\left\langle\boldsymbol{t}_{m}(\boldsymbol{r}), \mathcal{K}_{1}\left\{\boldsymbol{b}_{n}(\boldsymbol{r})\right\}\right\rangle \\
& +\eta_{1} \eta_{2}\left\langle\boldsymbol{t}_{m}(\boldsymbol{r}), \mathcal{K}_{2}\left\{\boldsymbol{b}_{n}(\boldsymbol{r})\right\}\right\rangle \\
\overline{\boldsymbol{Z}}_{22}^{T}[m, n]= & \eta_{2}\left\langle\boldsymbol{t}_{m}(\boldsymbol{r}), \mathcal{T}_{1}\left\{\boldsymbol{b}_{n}(\boldsymbol{r})\right\}\right\rangle \\
& +\eta_{1}\left\langle\boldsymbol{t}_{m}(\boldsymbol{r}), \mathcal{T}_{2}\left\{\boldsymbol{b}_{n}(\boldsymbol{r})\right\}\right\rangle
\end{aligned}
$$

and

$$
\begin{aligned}
\overline{\boldsymbol{Y}}_{11}^{T}[m, n]= & \eta_{1}\left\langle\boldsymbol{t}_{m}(\boldsymbol{r}), \mathcal{T}_{1}\left\{\boldsymbol{b}_{n}(\boldsymbol{r})\right\}\right\rangle \\
& -\eta_{2}\left\langle\boldsymbol{t}_{m}(\boldsymbol{r}), \mathcal{T}_{2}\left\{\boldsymbol{b}_{n}(\boldsymbol{r})\right\}\right\rangle \\
\overline{\boldsymbol{Y}}_{12}^{T}[m, n]= & -\left\langle\boldsymbol{t}_{m}(\boldsymbol{r}), \mathcal{K}_{1}\left\{\boldsymbol{b}_{n}(\boldsymbol{r})\right\}\right\rangle \\
& +\left\langle\boldsymbol{t}_{m}(\boldsymbol{r}), \mathcal{K}_{2}\left\{\boldsymbol{b}_{n}(\boldsymbol{r})\right\}\right\rangle \\
\overline{\boldsymbol{Y}}_{21}^{T}[m, n]= & \eta_{1} \eta_{2}\left\langle\boldsymbol{t}_{m}(\boldsymbol{r}), \mathcal{K}_{1}\left\{\boldsymbol{b}_{n}(\boldsymbol{r})\right\}\right\rangle \\
& -\eta_{1} \eta_{2}\left\langle\boldsymbol{t}_{m}(\boldsymbol{r}), \mathcal{K}_{2}\left\{\boldsymbol{b}_{n}(\boldsymbol{r})\right\}\right\rangle \\
\overline{\boldsymbol{Y}}_{22}^{T}[m, n]= & \eta_{2}\left\langle\boldsymbol{t}_{m}(\boldsymbol{r}), \mathcal{T}_{1}\left\{\boldsymbol{b}_{n}(\boldsymbol{r})\right\}\right\rangle \\
& -\eta_{1}\left\langle\boldsymbol{t}_{m}(\boldsymbol{r}), \mathcal{T}_{2}\left\{\boldsymbol{b}_{n}(\boldsymbol{r})\right\}\right\rangle .
\end{aligned}
$$

For the solution of a scattering problem, S-CNF provides a better-conditioned matrix equation compared to S-CTF. This is due to the well-tested identity terms on the diagonal blocks of the discretized S-CNF in (26). However, for the same discretization of the problem, S-CNF results are significantly inaccurate compared to S-CTF. This inaccuracy, which we relate the the well-tested identity terms [18], is extensively investigated for the $\mathrm{N}$ formulations of perfectly-conducting objects and dielectric bodies with moderate contrasts [5], [13]-[17]. It was shown that the accuracy of the $\mathrm{N}$ formulations can be improved to the levels of the $\mathrm{T}$ formulations by employing the linear-linear (LL) basis functions instead of the RWG functions [5], [16], [17]. MFIE inaccuracy can be reduced by using higher-order basis functions. Regularizing the $\mathrm{N}$ formulation by smoothing the identity terms is another method to improve accuracy; this has been proposed for 2-D problems [18].

An important issue for the stable formulations in (26) and (31) is to calculate the coefficients $\boldsymbol{x}^{i}$ and $\boldsymbol{y}^{i}$ for expanding the incident fields. For both S-CTF and S-CNF, we perform this by iteratively solving the sparse matrix equation

$$
\left[\begin{array}{cc}
\overline{\boldsymbol{I}} & 0 \\
0 & \overline{\boldsymbol{I}}
\end{array}\right] \cdot\left[\begin{array}{l}
\boldsymbol{x}^{i} \\
\boldsymbol{y}^{i}
\end{array}\right]=\left[\begin{array}{c}
\left\langle\boldsymbol{t}_{m}(\boldsymbol{r}), \hat{\boldsymbol{n}} \times \boldsymbol{H}^{i}(\boldsymbol{r})\right\rangle_{m=1,2, \ldots, N} \\
\left\langle\boldsymbol{t}_{m}(\boldsymbol{r}),-\hat{\boldsymbol{n}} \times \boldsymbol{E}^{i}(\boldsymbol{r})\right\rangle_{m=1,2, \ldots, N}
\end{array}\right]
$$

which usually requires negligible time compared to the iterative solutions of the main equations in (26) and (31) since $\overline{\boldsymbol{I}}$ is a highly sparse matrix, as defined in (30). However, the presence of the discretized identity operators in (40) deteriorates the accuracy of the results. Although this is not critical 
for S-CNF, which already contains identity operators, the accuracy of S-CTF can be significantly affected. Therefore, to obtain more accurate results, we propose a double-stabilized CTF (DS-CTF) based on calculating the coefficients related to the incident fields by solving the discretized form of (20), i.e.,

$$
\begin{aligned}
{\left[\begin{array}{cc}
\eta_{1} \overline{\boldsymbol{T}}_{1} & -\overline{\boldsymbol{K}}_{1} \\
\overline{\boldsymbol{K}}_{1} & \eta_{1}^{-1} \overline{\boldsymbol{T}}_{1}
\end{array}\right] } & \cdot\left[\begin{array}{c}
\boldsymbol{x}^{i} \\
\boldsymbol{y}^{i}
\end{array}\right] \\
& =-0.5\left[\begin{array}{c}
\left\langle\boldsymbol{t}_{m}(\boldsymbol{r}), \boldsymbol{E}^{i}(\boldsymbol{r})\right\rangle_{m=1, \ldots, N} \\
\left\langle\boldsymbol{t}_{m}(\boldsymbol{r}), \boldsymbol{H}^{i}(\boldsymbol{r})\right\rangle_{m=1, \ldots, N}
\end{array}\right]
\end{aligned}
$$

where

$$
\begin{aligned}
\overline{\boldsymbol{T}}_{1}[m, n] & =\left\langle\boldsymbol{t}_{m}(\boldsymbol{r}), \mathcal{T}_{1}\left\{\boldsymbol{b}_{n}(\boldsymbol{r})\right\}\right\rangle \\
\overline{\boldsymbol{K}}_{1}[m, n] & =\left\langle\boldsymbol{t}_{m}(\boldsymbol{r}), \mathcal{K}_{1}\left\{\boldsymbol{b}_{n}(\boldsymbol{r})\right\}\right\rangle .
\end{aligned}
$$

We note that the improved accuracy of DS-CTF comes at the cost of reduced efficiency since it is necessary to solve an additional dense matrix equation rather than the extremely sparse equation in (40).

\section{RESULTS}

First, we demonstrate the improved accuracy of the stable formulations in the solutions of scattering problems involving a small sphere of radius $0.5 \lambda_{1}$, where $\lambda_{1}$ is the wavelength outside the sphere. The sphere is located in free space and illuminated by a plane wave propagating in the $z$ direction with the electric field polarized in the $x$ direction. Discretization of the surface with $\lambda_{1} / 10$ mesh size produces 620 triangles and $2 \times 930$ RWG functions are employed to expand the unknown surface current densities. Matrix equations obtained by using conventional and stable formulations are solved iteratively. Elements of the matrix equations (interactions) are computed by numerical integration with $10^{-2}$ relative error. Fig. 1(a) shows the relative root-mean-square (RMS) error in the computed values of the far-zone electric field on the $\phi=0$ plane with respect to different contrasts $\left(\epsilon_{r}-1\right.$, where $\epsilon_{r}$ is the relative permittivity of the sphere) from $10^{-4}$ to $10^{-1}$. We consider

$$
f[j]=\lim _{r \rightarrow \infty}\left\{r E_{\theta}\left(r, \theta_{j}, \phi=0\right)\right\}
$$

where $r$ is the distance from the origin, $\theta_{j}=(j-1) \pi / 360$ for $j=1,2, \ldots, 360$, and $E_{\theta}$ is the $\theta$ component of the scattered electric field. We compute (44) by using different integral-equation formulations presented in this paper and also by the Mie-series method as a reference. Then, the RMS error is defined as

$$
e_{\mathrm{RMS}}=\frac{\left\|\boldsymbol{f}_{C}-\boldsymbol{f}_{A}\right\|_{2}}{\left\|\boldsymbol{f}_{A}\right\|_{2}}
$$

where $f_{C}$ and $f_{A}$ are the computational and analytical values, respectively, and $\|\cdot\|_{2}$ represents the 2-norm of the arrays involving 360 elements. Fig. 1(a) shows that CTF and CNF formulations encounter stability problems as contrast decreases and they fail to provide accurate results for low-contrast levels. With stable formulations, however, accuracy does not depend on the contrast and we obtain accurate results even for $\epsilon_{r}=1+10^{-4}$.

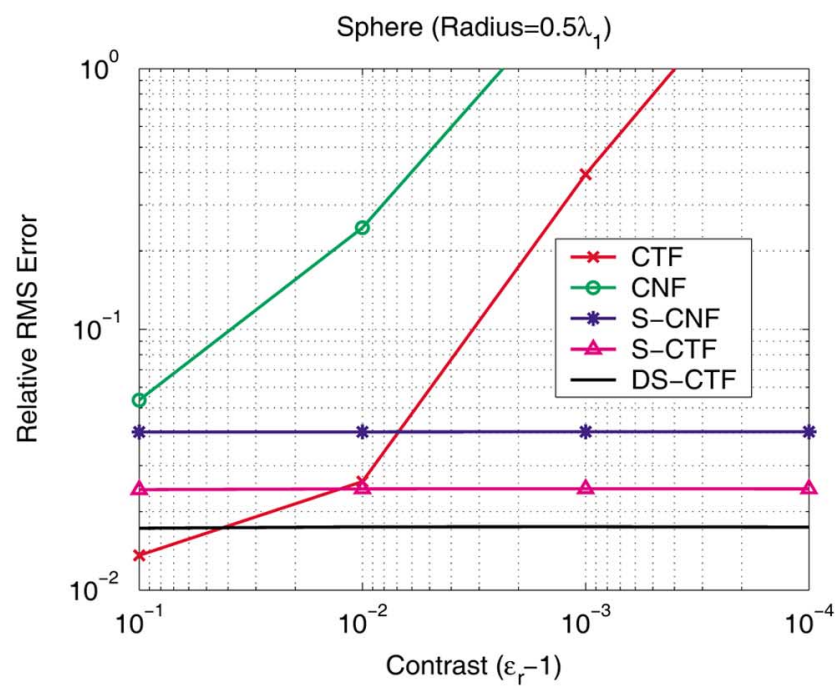

(a)

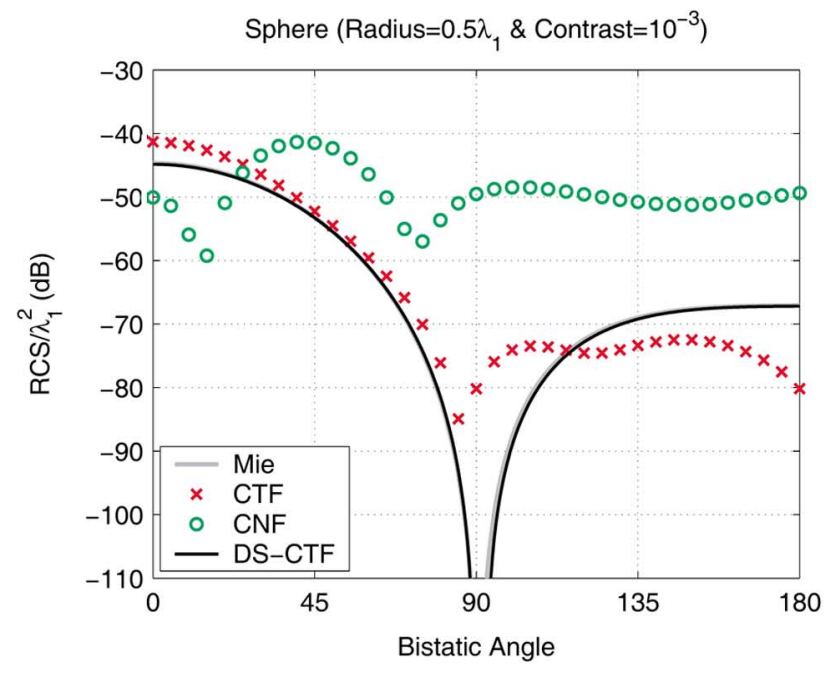

(b)

Fig. 1. (a) Root-mean-square (RMS) error in the solution of a scattering problem involving a sphere of radius $0.5 \lambda_{1}$ in free space. (b) Normalized bistatic RCS (RCS $/ \lambda_{1}^{2}$ ) of a sphere of radius $0.5 \lambda_{1}$ and relative permittivity $1+10^{-3}$ in free space.

We also observe that S-CTF is more accurate than S-CNF, as discussed in the previous section. In addition, DS-CTF provides the most accurate results for low-contrast problems, since its implementation is completely free of the identity terms. Fig. 1(b) presents the bistatic radar cross section (RCS) of the sphere when the contrast is $10^{-3}$. Normalized RCS $\left(\operatorname{RCS} / \lambda_{1}^{2}\right)$ is plotted in decibels $(\mathrm{dB})$ on the $\phi=0$ plane as a function of the observation angle, where $0^{\circ}$ corresponds to the forward-scattering direction. We observe that the traditional formulations are inaccurate, while the values obtained by DS-CTF are in agreement with the reference Mie-series solution.

For the solutions of larger dielectric problems, we employ MLFMA to accelerate the matrix-vector multiplications required by the iterative solver. Fig. 2 shows the solution of a scattering problem involving a sphere of radius $6 \lambda_{1}$ discretized with 264006 unknowns. In Fig. 2(a), we again consider the 


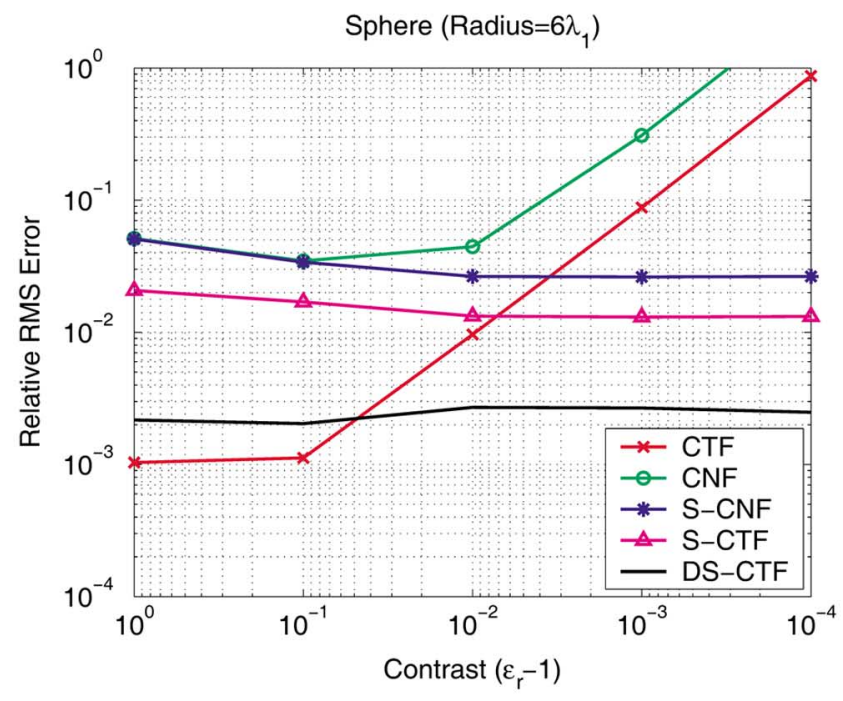

(a)

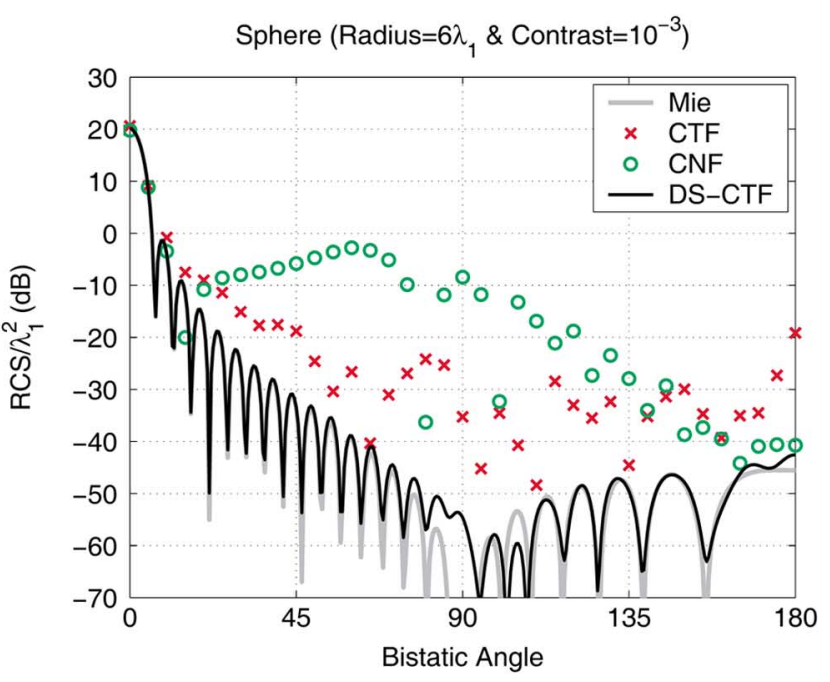

(b)

Fig. 2. (a) Root-mean-square (RMS) error in the solution of a scattering problem involving a sphere of radius $6 \lambda_{1}$ in free space. (b) Normalized bistatic $\operatorname{RCS}\left(\operatorname{RCS} / \lambda_{1}^{2}\right)$ of a sphere of radius $6 \lambda_{1}$ and relative permittivity $1+10^{-3}$ in free space.

relative RMS error for different formulations with respect to the contrast of the sphere. In the MLFMA implementation, near-field interactions are calculated by numerical integration with $10^{-2}$ error and far-field interactions are computed by multipole expansion with 3 digits of accuracy. Similar to the previous example, stable formulations do not break down as contrast decreases. In Fig. 2(b), bistatic RCS values (in dB) are plotted as a function of the observation angle when the contrast is $10^{-3}$. The traditional formulations are accurate only in the forward-scattering direction $\left(0^{\circ}\right)$, while the accuracy is improved significantly by using DS-CTF.

For the solution of the scattering problem involving a sphere of radius $6 \lambda$ with 0.1 contrast, Table I lists the iteration counts of the conjugate-gradient-squared (CGS) method to reduce the residual error to less than $10^{-6}$. We note that the extra costs of S-CTF and S-CNF are negligible compared to CTF and CNF,
TABLE I

NUMBERS OF CGS ITERATIONS FOR 264 006-UNKNOWN SPHERE PROBLEM $\left(10^{-6}\right.$ RESIDUAL ERROR)

\begin{tabular}{|l|c|c|c|c|c|}
\hline & CNF & CTF & S-CNF & S-CTF & DS-CTF \\
\hline Full System & 35 & 638 & 33 & 655 & $616+670$ \\
\hline Sparse System & - & - & 8 & 9 & - \\
\hline
\end{tabular}

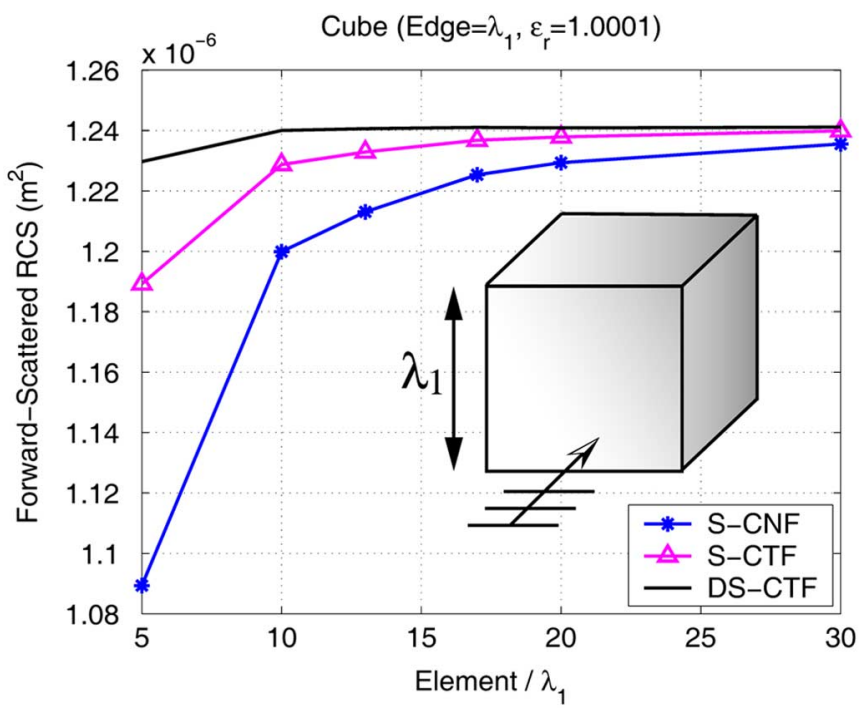

Fig. 3. Forward-scattered RCS $\left(\mathrm{m}^{2}\right)$ for a cube of edges $\lambda_{1}$ and relative permittivity $\epsilon_{r}=1.0001$ located in free space with respect to various discretizations of the scattering problem.

respectively. These stable formulations require only 8-9 iterations to solve the extremely sparse matrix equation in (40). On the other hand, using DS-CTF doubles the processing time compared to S-CTF since the solution of the dense matrix-equation in (41) requires 670 iterations, in addition to the 616 iterations for the solution of the main equation in (31). Table I also shows that both conventional and stable $\mathrm{T}$ formulations (CTF, S-CTF, and DS-CTF) require more iterations than $\mathrm{N}$ formulations (CNF and S-CNF). However, $\mathrm{T}$ formulations are generally more accurate to calculate the scattered fields compared to $\mathrm{N}$ formulations. As also discussed in Section III, this trade-off between the accuracy of the $\mathrm{T}$ formulations and the efficiency of the $\mathrm{N}$ formulations is commonly observed in various studies on integral-equation formulations [5], [13]-[17].

Finally, Fig. 3 presents the results of a scattering problem involving a cube with edges of 1 meter illuminated by a plane wave at $300 \mathrm{MHz}$. The cube has a relative permittivity of 1.0001 and it is located in free space. Fig. 3 shows the forward-scattered RCS values $\left(\mathrm{m}^{2}\right)$ for different discretizations of the geometry. Triangulation of the geometry with $\lambda_{1} / 5$ and $\lambda_{1} / 30$ mesh sizes leads to 1008 and 36756 unknowns, respectively. In the MLFMA implementation, near-field interactions are again calculated with $10^{-2}$ error and far-field interactions are computed with 3 digits of accuracy. Fig. 3 indicates that all stable formulations tend to converge to a limit (correct) value as the discretization becomes denser. However, convergence characteristics depend on the formulation type. We observe that S-CNF and DS-CTF have the slowest and the fastest convergence rates, respectively. Another interpretation of Fig. 3 is that the most 
accurate results are provided by DS-CTF for a given mesh size. Specifically, for the conventional $\lambda_{1} / 10$ triangulation (10 elements per $\lambda_{1}$ ), the error of $\mathrm{S}-\mathrm{CNF}$ is more than $3 \%$; this may not be acceptable since the elements of the matrix equation are calculated with at most $1 \%$ error.

\section{CONCLUSION}

In this paper, we report accurate solutions for scattering problems involving low-contrast dielectric objects. As the contrast decreases, traditional forms of the surface dielectric formulations break down. Therefore, we obtain accurate solutions by stabilizing the traditional forms of the formulations. In addition, we present various stabilization schemes and systematically reduce the error by eliminating the identity terms from the matrix equations. Considering a trade-off between efficiency and accuracy, the choice of which stabilization is preferable will depend on the application.

\section{APPENDIX}

To derive the identity in (20), we consider a "phantom" object with zero contrast. Then, using $\{a, b, c, d\}=\left\{\eta_{1}, \eta_{1}, \eta_{1}, \eta_{1}\right\}$ in (5) leads to

or

$$
\hat{\boldsymbol{t}} \cdot\left[\begin{array}{cc}
2 \eta_{1} \mathcal{T}_{1} & -2 \mathcal{K}_{1} \\
2 \eta_{1}^{2} \mathcal{K}_{1} & 2 \eta_{1} \mathcal{T}_{1}
\end{array}\right] \cdot\left[\begin{array}{c}
\boldsymbol{J}(\boldsymbol{r}) \\
\boldsymbol{M}(\boldsymbol{r})
\end{array}\right]=-\hat{\boldsymbol{t}} \cdot\left[\begin{array}{c}
\boldsymbol{E}^{i}(\boldsymbol{r}) \\
\eta_{1}^{2} \boldsymbol{H}^{i}(\boldsymbol{r})
\end{array}\right]
$$

$$
\hat{\boldsymbol{t}} \cdot\left[\begin{array}{cc}
\eta_{1} \mathcal{T}_{1} & -\mathcal{K}_{1} \\
\mathcal{K}_{1} & \eta_{1}^{-1} \mathcal{T}_{1}
\end{array}\right] \cdot\left[\begin{array}{c}
\boldsymbol{J}(\boldsymbol{r}) \\
\boldsymbol{M}(\boldsymbol{r})
\end{array}\right]=-0.5 \hat{\boldsymbol{t}} \cdot\left[\begin{array}{l}
\boldsymbol{E}^{i}(\boldsymbol{r}) \\
\boldsymbol{H}^{i}(\boldsymbol{r})
\end{array}\right] .
$$

Similarly, using $\{a, b, c, d\}=\{1,-1,1,-1\}$ in (11) leads to

$$
\begin{aligned}
& \hat{\boldsymbol{n}} \times\left[\begin{array}{cc}
2 \mathcal{K}_{1} & 2 \eta_{1}^{-1} \mathcal{T}_{1} \\
-2 \eta_{1} \mathcal{T}_{1} & 2 \mathcal{K}_{1}
\end{array}\right] \cdot\left[\begin{array}{c}
\boldsymbol{J}(\boldsymbol{r}) \\
\boldsymbol{M}(\boldsymbol{r})
\end{array}\right]=-\hat{\boldsymbol{n}} \times\left[\begin{array}{c}
\boldsymbol{H}^{i}(\boldsymbol{r}) \\
-\boldsymbol{E}^{i}(\boldsymbol{r})
\end{array}\right] \\
& \text { or } \\
& \hat{\boldsymbol{n}} \times\left[\begin{array}{cc}
\eta_{1} \mathcal{T}_{1} & -\mathcal{K}_{1} \\
\mathcal{K}_{1} & \eta_{1}^{-1} \mathcal{T}_{1}
\end{array}\right] \cdot\left[\begin{array}{c}
\boldsymbol{J}(\boldsymbol{r}) \\
\boldsymbol{M}(\boldsymbol{r})
\end{array}\right]=-0.5 \hat{\boldsymbol{n}} \times\left[\begin{array}{c}
\boldsymbol{E}^{i}(\boldsymbol{r}) \\
\boldsymbol{H}^{i}(\boldsymbol{r})
\end{array}\right] .
\end{aligned}
$$

Since an actual scatterer is not present, no scattered field is produced. Therefore, the total field is equal to the incident field everywhere and we can substitute

$$
\begin{aligned}
\boldsymbol{J}(\boldsymbol{r}) & =\hat{\boldsymbol{n}} \times \boldsymbol{H}(\boldsymbol{r})=\hat{\boldsymbol{n}} \times \boldsymbol{H}^{i}(\boldsymbol{r}) \\
\boldsymbol{M}(\boldsymbol{r}) & =-\hat{\boldsymbol{n}} \times \boldsymbol{E}(\boldsymbol{r})=-\hat{\boldsymbol{n}} \times \boldsymbol{E}^{i}(\boldsymbol{r})
\end{aligned}
$$

in (47) and (49) to obtain

$$
\begin{aligned}
\left\{\begin{array}{c}
\hat{\boldsymbol{t}} \cdot \\
\hat{\boldsymbol{n}} \times
\end{array}\right\}\left[\begin{array}{cc}
\eta_{1} \mathcal{T}_{1} & -\mathcal{K}_{1} \\
\mathcal{K}_{1} & \eta_{1}^{-1} \mathcal{T}_{1}
\end{array}\right] & {\left[\begin{array}{c}
\hat{\boldsymbol{n}} \times \boldsymbol{H}^{i}(\boldsymbol{r}) \\
-\hat{\boldsymbol{n}} \times \boldsymbol{E}^{i}(\boldsymbol{r})
\end{array}\right] } \\
& =-0.5\left\{\begin{array}{c}
\hat{\boldsymbol{t}} \\
\hat{\boldsymbol{n}} \times
\end{array}\right\}\left[\begin{array}{c}
\boldsymbol{E}^{i}(\boldsymbol{r}) \\
\boldsymbol{H}^{i}(\boldsymbol{r})
\end{array}\right] .
\end{aligned}
$$

\section{REFERENCES}

[1] A. J. Poggio and E. K. Miller, "Integral equation solutions of three-dimensional scattering problems," in Computer Techniques for Electromagnetics, R. Mittra, Ed. Oxford, U.K.: Pergamon Press, 1973, ch. 4.
[2] J. R. Mautz and R. F. Harrington, "Electromagnetic scattering from a homogeneous material body of revolution," $A E \ddot{U}$, vol. 33 , pp. 71-80, Feb. 1979.

[3] K. Umashankar, A. Taflove, and S. M. Rao, "Electromagnetic scattering by arbitrary shaped three-dimensional homogeneous lossy dielectric objects," IEEE Trans. Antennas Propag., vol. AP-34, no. 6, pp. 758-766, Jun. 1986.

[4] X.-Q. Sheng, J.-M. Jin, J. Song, W. C. Chew, and C.-C. Lu, "Solution of combined-field integral equation using multilevel fast multipole algorithm for scattering by homogeneous bodies," IEEE Trans. Antennas Propag., vol. 46, no. 11, pp. 1718-1726, Nov. 1998.

[5] P. Ylä-Oijala, M. Taskinen, and S. Järvenpää, "Surface integral equation formulations for solving electromagnetic scattering problems with iterative methods," Radio Sci., vol. 40, Nov. 2005, RS6002, doi:10. 1029/2004RS003169.

[6] T. W. Lloyd, J. M. Song, and M. Yang, "Numerical study of surface integral formulations for low-contrast objects," IEEE Antennas Wireless Propag. Lett., vol. 4, pp. 482-485, 2005.

[7] M. Hammer, D. Schweitzer, B. Michel, E. Thamm, and A. Kolb, "Single scattering by red blood cells," Appl. Opt., vol. 37, no. 31, pp. 7410-7418, Nov. 1998.

[8] D. A. Hill, "Electromagnetic scattering by buried objects of low contrast," IEEE Trans. Geosci. Remote Sen., vol. 26, no. 2, pp. 195-203, Mar. 1988.

[9] E. S. Thiele, "Scattering of electromagnetic radiation by complex microstructures in the resonant regime," Ph.D. dissertation, Univ. Pennsylvania, Philadelphia, 1998.

[10] P. Loschialpo, D. W. Forester, and J. Schelleng, "Anomalous transmission through near unit index contrast dielectric photonic crystals," J. Appl. Phys., vol. 86, no. 10, pp. 5342-5347, Nov. 1999.

[11] P. M. Goggans and A. Glisson, "A surface integral equation formulation for low contrast scatterers based on radiation currents," ACES J., vol. 10, pp. 15-18, Mar. 1995.

[12] S. M. Rao, D. R. Wilton, and A. W. Glisson, "Electromagnetic scattering by surfaces of arbitrary shape," IEEE Trans. Antennas Propag., vol. AP-30, no. 3, pp. 409-418, May 1982.

[13] E. Ubeda and J. M. Rius, "MFIE MOM-formulation with curl-conforming basis functions and accurate kernel integration in the analysis of perfectly conducting sharp-edged objects," Microw. Opt. Technol. Lett., vol. 44, no. 4, pp. 354-358, Feb. 2005.

[14] E. Ubeda and J. M. Rius, "Novel monopolar MFIE MoM-discretization for the scattering analysis of small objects," IEEE Trans. Antennas Propag., vol. 54, no. 1, pp. 50-57, Jan. 2006.

[15] Ö. Ergül and L. Gürel, "The use of curl-conforming basis functions for the magnetic-field integral equation," IEEE Trans. Antennas Propag., vol. 54, no. 7, pp. 1917-1926, July 2006.

[16] Ö. Ergül and L. Gürel, "Improving the accuracy of the magnetic field integral equation with the linear-linear basis functions," Radio Sci., vol. 41, 2006, RS4004, doi:10.1029/2005RS003307.

[17] Ö. Ergül and L. Gürel, "Linear-linear basis functions for MLFMA solutions of magnetic-field and combined-field integral equations," IEEE Trans. Antennas Propag., vol. 55, no. 4, pp. 1103-1110, Apr. 2007.

[18] C. P. Davis and K. F. Warnick, "High-order convergence with a low-order discretization of the 2-D MFIE," IEEE Antennas Wireless Propag. Lett., vol. 3, pp. 355-358, 2004.

[19] P. Ylä-Oijala and M. Taskinen, "Well-conditioned Müller formulation for electromagnetic scattering by dielectric objects," IEEE Trans. Antennas Propag., vol. 53, no. 10, pp. 3316-3323, Oct. 2005.

[20] Ö. Ergül and L. Gürel, "Improving the accuracy of the surface integral equations for low-contrast dielectric scatterers," in Proc. IEEE Antennas and Propagation Soc. Int. Symp., 2007, pp. 4853-4856.

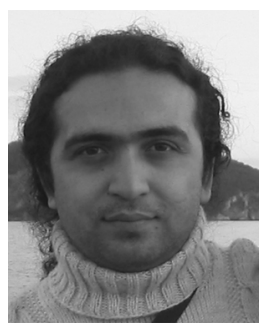

Özgür Ergül (S'98) was born in Yozgat, Turkey, in 1978. He received the B.Sc. and M.S. degrees in electrical and electronics engineering from Bilkent University, Ankara, Turkey, in 2001 and 2003, respectively, where he is currently working toward the Ph.D. degree.

Since 2001, he has served as a Teaching and Research Assistant in the Department of Electrical and Electronics Engineering at Bilkent University. He has been affiliated with the Computational Electromagnetics Group at Bilkent University from 2000 to 2005 and with the Computational Electromagnetics Research Center (BiLCEM) since 2005. His research interests include fast and accurate algorithms for the solutions of large and complicated structures, integral equations, parallel programming, and iterative techniques.

Mr. Ergül is a recipient of the 2007 IEEE Antennas and Propagation Society Graduate Fellowship and the 2007 Leopold. B. Felsen Award for Excellence 
in Electrodynamics. He is the Secretary of Commission E (Electromagnetic Noise and Interference) of the URSI Turkey National Committee. His academic endeavors are supported by the Scientific and Technical Research Council of Turkey (TUBITAK) through a Ph.D. scholarship.

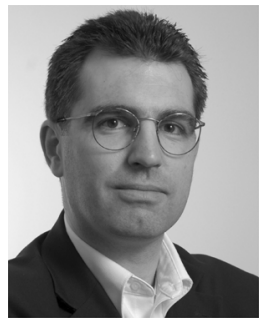

Levent Gürel (S'87-M'92-SM'97) received the B.Sc. degree from the Middle East Technical University (METU), Ankara, Turkey, in 1986, and the M.S. and $\mathrm{Ph} . \mathrm{D}$. degrees from the University of Illinois at Urbana-Champaign (UIUC), in 1988 and 1991, respectively, all in electrical engineering.

He joined the Thomas J. Watson Research Center of the International Business Machines Corporation, Yorktown Heights, NY, in 1991, where he worked as a Research Staff Member on the electromagnetic compatibility (EMC) problems related to electronic packaging, on the use of microwave processes in the manufacturing and testing of electronic circuits, and on the development of fast solvers for interconnect modeling. Since 1994, he has been a faculty member in the Department of Electrical and Electronics Engineering of the Bilkent University, Ankara, where he is currently a Professor. He was a Visiting Associate Professor at the Center for Computational Electromagnetics (CCEM) of the UIUC for one semester in 1997. He returned to the UIUC as a Visiting Professor in 2003-2005, and as an Adjunct Professor after 2005. He founded the Computational Electromagnetics
Research Center (BiLCEM) at Bilkent University in 2005, where he is serving as the Director. His research interests include the development of fast algorithms for computational electromagnetics (CEM) and the application thereof to scattering and radiation problems involving large and complicated scatterers, antennas and radars, frequency-selective surfaces, high-speed electronic circuits, optical and imaging systems, nanostructures, and metamaterials. He is also interested in the theoretical and computational aspects of electromagnetic compatibility and interference analyses. Ground penetrating radars and other subsurface scattering applications are also among his research interests. Since 2006, his research group has been breaking several world records by solving extremely large integral-equation problems, the largest involving as many as 85 million unknowns.

Prof. Gürel has received much recognition for his accomplishments, including an award from the Turkish Academy of Sciences (TUBA) in 2002 and the Scientific and Technical Research Council of Turkey (TUBITAK) in 2003. He served as the Chairman of the AP/MTT/ED/EMC Chapter of the IEEE Turkey Section in 2000-2003. He founded the IEEE EMC Chapter in Turkey in 2000. He served as the Co-Chairman of the 2003 IEEE International Symposium on Electromagnetic Compatibility. He is a member of the General Assembly of the European Microwave Association, a member of the USNC of the International Union of Radio Science (URSI), and the Chairman of Commission E (Electromagnetic Noise and Interference) of URSI Turkey National Committee. He organized and served as the General Chair of the CEM'07 Computational Electromagnetics International Workshop in 2007. $\mathrm{He}$ is currently serving as Associate Editor of Radio Science and the IEEE ANTENNAS AND WIRELESS PROPAGATION LETTERS. 